\title{
Deep Mediatization during COVID-19: An Interview with Professor Andreas Hepp, University of Bremen
}

\author{
SANTHOSH KUMAR PUTTA, Osmania University \\ BISSIE ANDERSON, University of Stirling \\ Co-Editors
}

[SKP and BA] Professor Hepp, your seminal theoretical work (individual and in collaboration with Nick Couldry) on mediatization has sought to understand the increasingly mediated construction of reality in today's digitized world. Your book Deep Mediatization (Routledge, 2020) argues that mediatization has reached an advanced stage in which all elements in our social world are deeply related to the media and other technological infrastructures. Has this process accelerated and deepened further with the COVID-19 pandemic?

[AH] Certainly, it has - in a sense, we can say that we have experienced the pandemic as deeply mediatized. At least four points can be identified here. First, from the initial outbreak, we have experienced the pandemic on the basis of expectations having been mediated. Films and series featuring dangerous viruses, their rapid spread and humanity's struggle with them have been popular since at least the 1970s. And for those who did not already know them, they were readily available through the various digital platforms after Covid-19 broke out. With this in mind, we approached the pandemic from existing media-mediated scripts on what 'can happen' and 'how to deal with it'. Second, we have a mediated experience of the pandemic itself: what we know about the pandemic has been communicated to us through the media, and here partially automated data journalism - the continuous visual processing of the latest Corona figures (infections, deaths, vaccinations) - has, at times, played a significant role. Third, we are dealing with an ongoing media-mediated analysis of the course the pandemic takes. What is meant here is that digital media and their infrastructures in particular are being used to obtain 'data' on the ways in which people are dealing with the pandemic. Examples of this include the analysis of people's mobility during the lockdown using login data from their mobile phones or a range of mathematical models of possible pandemic outcomes using various other digital data. Fourth and finally, we were repeatedly confronted with the idea of a media-based 'solution' to individual problems brought up by the pandemic. Digital media in particular should be mentioned here, for example, when at the beginning of the pandemic politicians imagined a Covid app as the central solution strategy, digital platforms were seen as the solution to prevent a collapse of the local economy and cultural industries, or when working from home was only possible through specific platforms and video conferencing systems. In all these cases, significant facets of the 'solutions' were bought from Silicon Valley and made these companies money as individuals became poorer as a result of the pandemic. Inequalities of the emerging digital society were probably made clear to all by the pandemic and in all likelihood were further exacerbated. 
[SKP and BA] To what extent does the concept deep mediatization relate to technological determinism? Is deep mediatization a phenomenon that solely results from advancements in information and communication technologies, or is it determined by other factors too?

[AH] The mediatization approach has nothing to do with technological determinism, on the contrary: it always assumes that media technologies, like all technologies, are to be understood as part of culture and society. That is why it is argued that the focus should be on the interrelationship between media and communication on the one hand and culture and society on the other - or formulated differently: There is a need to focus on how we produce certain forms of culture and society by means of certain media technologies, which always remain part of culture and society. Here the position is very clear, it is not about 'effect' but about complex interrelations and dynamics. That said, we should keep in mind that certain forms of culture and society are inconceivable without certain media technologies. And that is precisely why it remains relevant to ask how cultures and societies change with media, which is different from asking what the effects of media technologies are.

[SKP and BA] In your book you write about the role of supra-individual actors in the creation of deep mediatization. Who are these key actors and what responsibility do they bear for constructing our mediated reality?

[AH] When we look at the process of deep mediatization, there are two supra-individual actors in particular who are always in the foreground: Private companies and state agencies. These are certainly important drivers of deep mediatization. This process is human-made and, therefore, economic and political interests play a large role. One can call these kinds of supra-individual actors 'corporate actors'. But there are also 'collective actors' who play an important role in the 'making' of deep mediatization. Mostly, the focus here has been on social movements, for example, the open-source movement and its notion of a society shaped by technology. My argument is that we should have in addition another collective actor in mind, namely pioneer communities. Examples of this are the Whole Earth Network in the San Francisco Bay Area studied by Fred Turner or the Quantified Self and Maker movements. These are a kind of hybrid between social movements and think tanks, have certain ideas of a future shaped by digital media, and are characterized by experimental practices to these ends. Only in rare cases does society change directly in the way the members of pioneer communities imagine it. But through their experimental practices and imaginations, they lay the ground for possible change. That is why they should be kept in mind.

[SKP and BA] Societies have been mediatized for a long time, now even more so with the use of algorithms and artificial intelligence. Do you think the acceleration of the process of mediatization we have experienced in the pandemic will eventually lead to something akin to hypermediatization? Will we see that happen in the future and, if so, what manifestations do you expect hypermediatization to take? 
$[\mathrm{AH}] \mathrm{I}$ wouldn't introduce another term here, but you certainly can. But what seems important to me in this context is an underlying argument: because media have become digital, we now, in relation to media, have tighter loops of recursivity than we had in in the not-too-distant past. We continuously leave digital traces on platforms through our practices, and these traces are in turn reflected back to us, not only on an individual level, but also on a collective level: as topics that 'trend', as 'recommendations' that follow from previous purchases, as 'collectivities' that form around platforms, and so on. Such processes happen almost in real time and are also the basis for the ongoing technological development of digital platforms. These tight loops of recursivity did not exist in this form before digital media and their infrastructures.

[SKP and BA] Addressing the challenges presented by deep mediatization to mediatization research, you argue that the latter must incorporate the analysis of algorithms, data, and digital infrastructures. Should corporate practices of developing and encouraging certain consumer behavior and user/audience engagement be part of this conceptualization?

[AH] Certainly, it is important to have a broad view and my references are not to be understood as being entirely complete. I wanted to point out one thing in particular: If communication and media research wants to take deep mediatization seriously, its former conceptual tools are not sufficient, nor are its methods. It must look for connections, for example, with critical data studies or computational social sciences, but without giving up its own focus. I am very reassured that this broadening of this perspective has increased in the last few years and that, for example, questions of technologies and algorithms have been given the space they should have.

[SKP and BA] Most of the social networking sites are free to use and owned by some the richest corporations in the world. Users of these platforms rarely give much thought to why these platforms are free and how corporations profit from them. Can the theory of deep mediatization be used to explain how consumers are fed certain information and conditioned for these tech giants' profit, while consumers perform what Christian Fuchs refers to as "digital labour"?

[AH] Yes, it certainly can. A key argument is to bring the basic ideas of Norbert Elias' process sociology into research on media and communication, and here it is the argument to understand media not as something static, but as an ongoing process of the institutionalization and the materialization of human practice. In this way, one can, and should, also ask the question: What forms of value creation are institutionalized? And how are these inscribed in the material structures of platforms?

[SKP and BA] You argue that there is an entanglement of general social practices with media and that the lines between communicative action and physical action are blurring. With the advancements and increased use of automated technologies, how important is media literacy? Do you think deep mediatization is something that is controllable? If so, how? 
[AH] Especially with deep mediatization, questions of media literacy become even more important than before. Above all, in my view, they no longer appear as something separate. In the age of legacy media - i.e. the classical mass media - media literacy was understood as the ability to critically comprehend media discourses and, in the best case, to participate in them. Nowadays, questions of media literacy permeate the entirety of everyday practice: How do I communicate with my friends through which medium? How do I handle my own data? Who do I allow access to, who not? What problematic dynamics can develop in online discourses? That said, deep mediatization is a term used to describe an overarching process of change, just like individualization or globalization. As such, it is not easily controllable by a single person. However, because it is made by us humans, we can always ask ourselves the question: Who is being excluded? Where does deep mediatization contribute to a 'good life' and where does it not? Asking such questions is not simply about individual media literacy, but fundamentally about organizing platforms and digital infrastructures.

[SKP and BA] Deep mediatization employs a functionalist approach to understanding the social construction of reality. By doing so, is it undermining individual subjectivity? Can the pervasive persuasive capabilities of new media be resisted to achieve individual autonomy? And what are the effects of a deeply mediatized world on the psychology and well-being of the individual?

[AH] From my point of view, an important basis for understanding deep mediatization is the aforementioned process sociology. This does not juxtapose the individual and society, but understands society as always emerging through individual practice and that individuals are always part of society. Norbert Elias coined the term 'figuration' to capture this. A community, for example, is a figuration of people who share certain practices and orientations of meaning. One can now look at this figuration from two perspectives: From the perspective of the figuration as a whole or from the perspective of the individual as part of that figuration. This is particularly helpful for questions of autonomy. Autonomy is never absolute, but relational: it always arises in certain figurations. And then the decisive question in relation to digital media and infrastructures is: What spaces for practice do they promote for people in certain figurations? Where do they replicate previous restrictions? Where do they create new ones? From my point of view, it is this kind of relational thinking that helps.

[SKP and BA] In your eponymous book, you argue that, considering deep mediatization the new normal, digital media and their infrastructures should be structured in such a way so as to enable "the good life". How can this be best achieved? What conditions need to be in place and whose responsibility is it to drive a more human-centred and ethical media ecosystem?

[AH] I have already hinted at this somewhat: I think it is important to look at the institutionalization and materialization of digital media as they currently exist and to ask ourselves the question: Are these what we really want? Digital platforms, for example, are currently almost all in the hands of private companies that use them to create value. If platforms have become so important for today's communication that we see them as infrastructure, 
shouldn't we organize the availability of them differently than we have done so far? For my part, it is important to first ask how we envision our media infrastructure in and across individual societies and then develop organizational models that correspond to these values. This is a multilayered political process, but I think it is worth going through.

[SKP and BA] At the ICA 2020 conference, which took place virtually for the first time due to the global pandemic, you and Wiebke Loosen argued that COVID-19 could be theorized as a "mediatized collective break" during which we are experiencing the "refiguration of public connection". Thinking of contingency relations, in terms of what has been, what we are going through and what will come after the pandemic, what transformations in public communications do you expect COVID-19 will lead to?

[AH] It is always difficult to predict the future, so I am cautions about this. Maybe it helps to explain a little what Wiebke Loosen and I meant by that: First of all, the pandemic was a break with previous patterns of media production, while at the same time the entire public discourse in many countries was suddenly focused on only one topic. In addition, digital forms of dissemination gained importance at all levels from one day to another. These kind of breaks in established patterns of production do two things: on the one hand, they are like a lens through which pre-existing problems become more visible. And in the case of digital media, these concerns include questions of inequality, questions of the quality of public discourse, and so on. Here, the pandemic has made certain problems very apparent to us. On the other hand, such a break always opens up possibilities for change. Some changes will simply be that people are more used to doing certain things through digital media than before. In this respect, the pandemic can further advance the process of deep mediatization. But at the same time, it has also shown that there are limits to digital mediation. And here we will be confronted with new processes of societal negotiation. What do we want to have mediated digitally? And what do we want to experience directly?

[SKP and BA] Finally, how does this Networking Knowledge special issue contribute to the debates on mediatization and the mediated construction of reality - both at a time of COVID-19 pandemic and more broadly?

[AH] I think the thematic issue is a very important contribution to furthering the discussion on deep mediatization. From my point of view, the various articles show very well what I said at the beginning of the interview, namely that we experienced the pandemic as deeply mediatized. This applies to the coverage of the pandemic, as Zheng Yang's contribution shows with the example of war metaphors in Chinese reporting; it applies to the intimate space of politicians, which Sara García Santamaría analyses; it is shown, as Jeannine Teichert explains, by the experience of close friendships or that of presence, as Anastasiya Maksymchuk and Katherina Radeva deal with it. I also found remarkable the negotiation of friendships and the closeness and distance in them, analyzed by Lauren Dempsey, and the Zen assembly as it took place online and is reflected by Lisette Torres. Merlin Seller looks at the role of digital games in lock-down situations and Donnalyn $\mathrm{Xu}$ or Morven Gow at the experience of navigating loneliness and intimacy in the digital space. All of us probably know something similar from our own experience of the 
pandemic. I think the important contribution the issue has to offer is to have made this all accessible for analytical reflection.

\section{Biographies}

Santhosh kumar Putta is a UGC - Junior Research Fellow at the Department of Communication and Journalism, UCASS, Osmania University, India.

Email: santoshkera@gmail.com

Bissie Anderson is a doctoral candidate in Journalism at the University of Stirling, Scotland. She was Editor-in-chief of Networking Knowledge in 2020.

Email: bissie.anderson@stir.ac.uk 